\title{
Predictions of Ligand Selectivity from Absolute Binding Free Energy Calculations
}

\author{
Matteo Aldeghi, ${ }^{\dagger \odot}$ Alexander Heifetz, ${ }^{\ddagger}$ Michael J. Bodkin, ${ }^{\ddagger}$ Stefan Knapp, ${ }^{\S, \|, \perp}$ and Philip C. Biggin ${ }^{*, \dagger}$ \\ ${ }^{\dagger}$ Structural Bioinformatics and Computational Biochemistry, Department of Biochemistry, University of Oxford, South Parks Road, \\ Oxford OX1 3QU, U.K. \\ ${ }^{\ddagger}$ Evotec (U.K.) Ltd., 114 Innovation Drive, Milton Park, Abingdon, Oxfordshire OX14 4RZ, U.K. \\ ${ }^{\S}$ Structural Genomics Consortium, Nuffield Department of Clinical Medicine, University of Oxford, Old Road Campus Research \\ Building, Roosevelt Drive, Oxford OX3 7DQ, U.K. \\ ${ }^{\|}$Target Discovery Institute, Nuffield Department of Clinical Medicine, University of Oxford, Roosevelt Drive, Oxford OX3 7BN, \\ U.K. \\ ${ }^{\perp}$ Institute for Pharmaceutical Chemistry, Goethe University Frankfurt, 60438 Frankfurt, Germany
}

Supporting Information

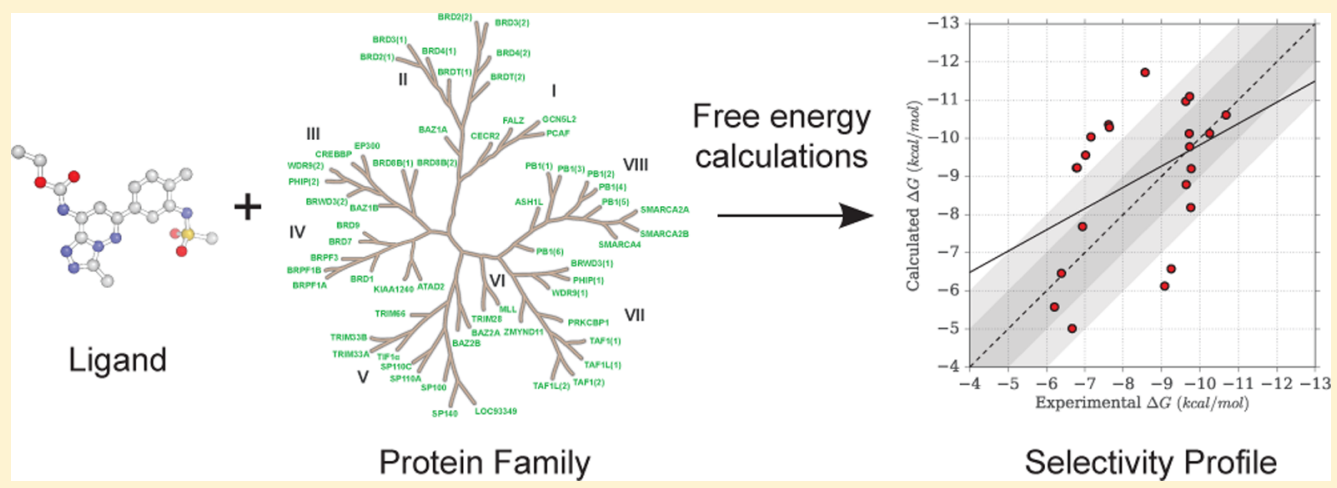

ABSTRACT: Binding selectivity is a requirement for the development of a safe drug, and it is a critical property for chemical probes used in preclinical target validation. Engineering selectivity adds considerable complexity to the rational design of new drugs, as it involves the optimization of multiple binding affinities. Computationally, the prediction of binding selectivity is a challenge, and generally applicable methodologies are still not available to the computational and medicinal chemistry communities. Absolute binding free energy calculations based on alchemical pathways provide a rigorous framework for affinity predictions and could thus offer a general approach to the problem. We evaluated the performance of free energy calculations based on molecular dynamics for the prediction of selectivity by estimating the affinity profile of three bromodomain inhibitors across multiple bromodomain families, and by comparing the results to isothermal titration calorimetry data. Two case studies were considered. In the first one, the affinities of two similar ligands for seven bromodomains were calculated and returned excellent agreement with experiment (mean unsigned error of $0.81 \mathrm{kcal} / \mathrm{mol}$ and Pearson correlation of 0.75 ). In this test case, we also show how the preferred binding orientation of a ligand for different proteins can be estimated via free energy calculations. In the second case, the affinities of a broad-spectrum inhibitor for 22 bromodomains were calculated and returned a more modest accuracy (mean unsigned error of $1.76 \mathrm{kcal} / \mathrm{mol}$ and Pearson correlation of 0.48 ); however, the reparametrization of a sulfonamide moiety improved the agreement with experiment.

\section{INTRODUCTION}

Binding selectivity is one of the most important requirements for the development of a safe therapeutic; in fact, large differences in binding affinity between the intended target and similar proteins are often sought in order to avoid unwanted and often

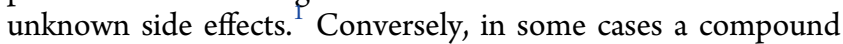
with a specific promiscuous profile might be equally desirable for reasons of efficacy. ${ }^{2,3}$ Yet, the identification and design of selective (or promiscuous) chemical tools is a difficult task, in particular when working on protein families with conserved folds and similar binding pocket residues. A typical example is provided by the protein kinase family, for which different assay panels have been developed in order to determine selectivity profiles of different ligands. ${ }^{4,5}$ The development of bromodomain (BRD) chemical probes poses the same selectivity challenge due their conserved fold. ${ }^{6}$ BRDs are epigenetic mark readers that specifically recognize $\varepsilon$ - $N$-lysine acetylation motifs; 61 human BRDs have been found in 46 human nuclear and cytoplasmic proteins, and they are typically divided into eight

Received: November 4, 2016

Published: December 23, 2016 
a

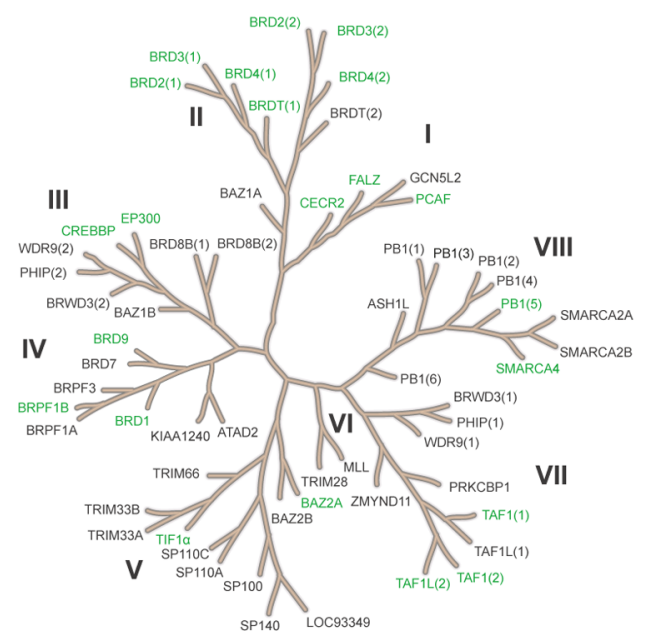

b

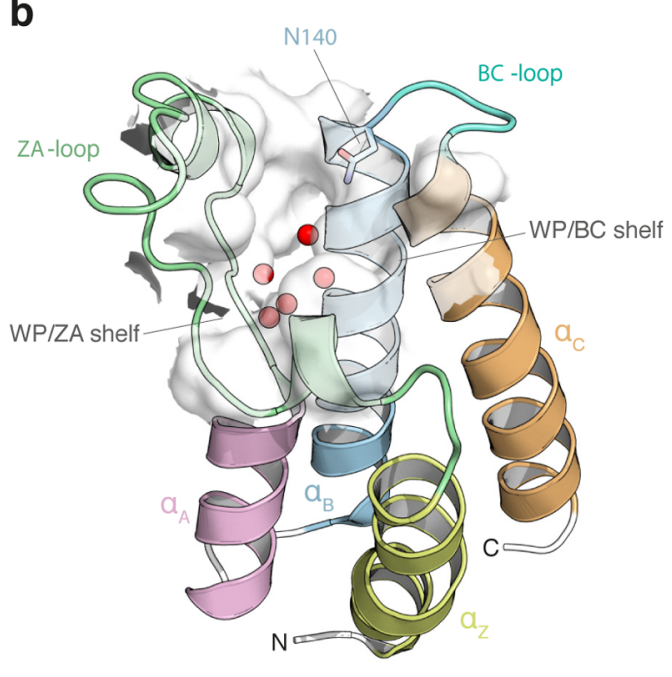

C<smiles>[R10][R20]#[R16]</smiles><smiles>[R12][R]([R1])O</smiles>

Bromosporine

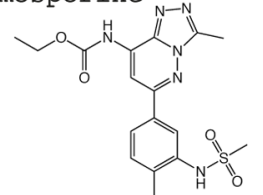

Figure 1. Ligands and proteins considered in this study. (a) Phylogenetic tree of the human bromodomain family; in green are the BRDs included in the study. (b) Conserved fold of human bromodomains (PDB ID 2OSS). Depicted as red spheres is the conserved water network found in most BRD binding pockets, and as blue sticks the conserved hydrogen bond donor, an asparagine residue in the majority of BRDs. A transparent surface shows the cavity in between the ZA and BC loops forming the acetyl-lysine binding pocket. Two clefts, placed in between the WP residues (conserved in subfamilies I and II) and the ZA and BC loops and exploited by many bromodomain inhibitors are indicated. (c) Chemical structures of the compounds.

families based on sequence and structural similarity (Figure 1a). ${ }^{7}$ With acetylation motifs often found in macromolecular complexes implicated in chromatin remodeling, DNA repair and cellcycle control, and especially on histones, BRD inhibitors are finding broad application in medicine and basic biological research, in particular in oncology and inflammation. Despite their sequence diversity, they all share a conserved fold (Figure 1b) that renders the design of selective ligands a challenging task. Selective probes can, however, lead to better preclinical target validation and profiling, and eventually to better clinical outcomes. ${ }^{6}$

Engineering selectivity adds considerable complexity to the rational design of new drugs, as it involves the optimization of multiple binding affinities. Different strategies have been adopted for selectivity design, such as shape and electrostatics complementarity, conformational selection, water displacement, and allosteric binding. Yet, engineering selectivity still remains a challenge for the drug design process. ${ }^{1}$ Computationally, the prediction of binding selectivity is a difficult task too, and not many generally applicable methodologies are available to the computational and medicinal chemistry communities. Henrich et $\mathrm{al}^{8}{ }^{8}$ evaluated the performance of target-specific scoring functions, developed from known sets of binders, for three protein targets: thrombin, trypsin, and urokinase-type plasminogen activator. The quality of the predictions appeared to be system dependent and the authors discussed how inclusion of protein flexibility and conserved waters might result in better docking methods. Thanks to advances in theory and computing, however, the prediction of binding affinities using physics-based biomolecular simulations is becoming increasingly feasible. Allatom simulations naturally take into account effects such as the dynamics of the binding pocket, the role of the solvent, and entropy changes upon binding, which are poorly captured with scoring-function-based approaches. ${ }^{8,9}$ In particular, free energy calculations based on molecular dynamics (MD) and alchemical pathways could offer an alternative and more rigorous approach to the problem, and one that does not require the use of training sets. Moreover, such calculations are becoming progressively more accessible to current levels of computational power. ${ }^{10,11}$
Recent studies have shown the potential of relative binding free energy (RBFE) calculations for small-molecule lead optimization. ${ }^{12-14}$ RBFE calculations can also allow the evaluation of free energy changes upon protein mutation, ${ }^{15-19}$ but they become unfeasible when a large number of residues differ between the proteins. Absolute binding free energy (ABFE) calculations are a more general approach as they do not require knowledge of any affinity value in advance and, in principle, could be used to compare the affinity of any ligand for any protein target. Such calculations could thus be employed as a tool to probe ligand selectivity across different binding pockets, in order to identify the scaffolds providing the desired selectivity profile. For instance, Lin and co-workers used absolute free energy calculations to explain the specificity of Gleevec for Abl kinase versus similar homologous tyrosine kinases. ${ }^{20-22}$ More recently, we have shown how calculations based on standard implementations of alchemical transformations might be applied for the accurate affinity estimation of diverse, drug-like organic molecules. $^{10}$

ABFE calculations are computationally demanding and, despite the few examples mentioned above, their performance has had only limited testing against experimental data, with a focus on model systems. ${ }^{23-26}$ Careful retrospective validation is therefore still needed before the methodology can be confidently applied in a prospective drug discovery scenario, with the ability to anticipate when these calculations might be the most suitable tool and where potential pitfalls might lie. Here, we test the robustness of $\mathrm{ABFE}$ calculations across different binding pockets, in order to assess the accuracy of the computational method against multiple proteins and thus evaluate its performance and potential utility for the study of ligand selectivity. We calculated the affinities for 36 complexes, involving 22 bromodomains and 3 ligands (RVX-OH, RVX-208, and bromosporine; see Figure 1c), without any information on the affinities or structures of the complexes. We compare the results with high-quality isothermal titration calorimetry (ITC) data and discuss the performance of the calculations in relation to their potential application in drug design. 
Table 1. Summary of the Calculation Results for RVX-OH and RVX-208, along with Information about the Experimental Structural and Affinity Data ${ }^{a}$

\begin{tabular}{|c|c|c|c|c|c|c|c|}
\hline \multirow[b]{2}{*}{ protein } & \multirow[b]{2}{*}{ ligand } & \multirow[b]{2}{*}{ PDB ID } & \multirow[b]{2}{*}{ pose } & \multirow[b]{2}{*}{$\Delta G_{\exp }(\mathrm{kcal} / \mathrm{mol})$} & \multicolumn{2}{|c|}{$\Delta G_{\text {calc }}(\mathrm{kcal} / \mathrm{mol})$} & \multirow[b]{2}{*}{$\Delta G_{\mathrm{calc}}-\Delta G_{\mathrm{exp}}(\mathrm{kcal} / \mathrm{mol})$} \\
\hline & & & & & H-pose & V-pose & \\
\hline $\mathrm{BRD} 2(1)$ & RVX-OH & 4ALG & $\mathrm{H}$ & $-8.5 \pm 0.1$ & $-9.8 \pm 0.1$ & $-6.5 \pm 0.1$ & $-1.3 \pm 0.2$ \\
\hline BRD3(1) & $\mathrm{RVX}-\mathrm{OH}$ & $3 S 91$ & $\mathrm{H}$ & $-8.0 \pm 0.1$ & $-6.7 \pm 0.1$ & $-5.7 \pm 0.1$ & $+1.3 \pm 0.2$ \\
\hline $\mathrm{BRD} 3(2)$ & $\mathrm{RVX}-\mathrm{OH}$ & $3 S 92$ & $\mathrm{~V}$ & $-8.8 \pm 0.1$ & $-6.6 \pm 0.2$ & $-7.4 \pm 0.1$ & $+1.5 \pm 0.2$ \\
\hline BRD4(1) & $\mathrm{RVX}-\mathrm{OH}$ & $2 \mathrm{OSS}$ & $\mathrm{H}$ & $-9.0 \pm 0.1$ & $-9.9 \pm 0.1$ & $-6.6 \pm 0.1$ & $-0.9 \pm 0.2$ \\
\hline $\operatorname{BRDT}(1)$ & $\mathrm{RVX}-\mathrm{OH}$ & $4 \mathrm{KCX}$ & $\mathrm{H}$ & $-7.7 \pm 0.1$ & $-7.7 \pm 0.1$ & $-6.3 \pm 0.1$ & $+0.0 \pm 0.2$ \\
\hline $\mathrm{BRD} 2(1)$ & RVX-208 & $4 \mathrm{ALG}$ & $\mathrm{V}$ & $-6.9 \pm 0.1$ & $\mathrm{n} / \mathrm{a}$ & $-7.2 \pm 0.1$ & $-0.3 \pm 0.2$ \\
\hline $\mathrm{BRD} 2(2)$ & RVX-208 & $4 \mathrm{MR} 5$ & $\mathrm{~V}$ & $-8.5 \pm 0.1$ & $\mathrm{n} / \mathrm{a}$ & $-7.5 \pm 0.1$ & $+0.9 \pm 0.2$ \\
\hline BRD3(1) & RVX-208 & $3 S 91$ & $\mathrm{~V}$ & $-7.1 \pm 0.1$ & $\mathrm{n} / \mathrm{a}$ & $-5.5 \pm 0.1$ & $+1.6 \pm 0.2$ \\
\hline BRD3(2) & RVX-208 & $3 S 92$ & $\mathrm{~V}$ & $-8.8 \pm 0.1$ & $\mathrm{n} / \mathrm{a}$ & $-8.7 \pm 0.1$ & $+0.1 \pm 0.2$ \\
\hline $\mathrm{BRD} 4(2)$ & RVX-208 & $2 \mathrm{OUO}$ & $\mathrm{V}$ & $-9.1 \pm 0.1$ & $\mathrm{n} / \mathrm{a}$ & $-9.7 \pm 0.1$ & $-0.6 \pm 0.2$ \\
\hline $\operatorname{BRDT}(1)$ & RVX-208 & $4 \mathrm{KCX}$ & $\mathrm{V}$ & $-7.0 \pm 0.1$ & $\mathrm{n} / \mathrm{a}$ & $-6.5 \pm 0.1$ & $+0.5 \pm 0.2$ \\
\hline \multicolumn{5}{|c|}{ mean unsigned error } & \multicolumn{3}{|c|}{$0.81[0.74,0.90] \mathrm{kcal} / \mathrm{mol}$} \\
\hline & \multicolumn{3}{|c|}{ root mean square error } & & \multicolumn{3}{|c|}{$0.95[0.87,1.05] \mathrm{kcal} / \mathrm{mol}$} \\
\hline & \multicolumn{3}{|c|}{$\% \leq 1.0 \mathrm{kcal} / \mathrm{mol}$} & & \multicolumn{3}{|c|}{$64[50,71]$} \\
\hline & \multicolumn{3}{|c|}{$\% \leq 2.0 \mathrm{kcal} / \mathrm{mol}$} & & \multicolumn{3}{|c|}{$100[100,100]$} \\
\hline & \multicolumn{3}{|c|}{ Pearson's $r$} & & \multicolumn{3}{|c|}{$0.75[0.67,0.80]$} \\
\hline & \multicolumn{3}{|c|}{ Spearman's $\rho$} & & \multicolumn{3}{|c|}{$0.78[0.64,0.85]$} \\
\hline
\end{tabular}

${ }^{a}$ ITC data were taken from Picaud et al. ${ }^{37}$ All uncertainties are one standard error of the mean. ITC uncertainties are an estimate based on the standard deviation of the $\Delta G$ values observed in the ABRF-MIRG'02 inter-laboratory assessment. ${ }^{38}$ Values for the difference $\Delta G_{\text {calc }}-\Delta G_{\text {exp }}$ may appear inconsistent due to rounding. "PDB ID" refers to RCSB Protein Data Bank code for the protein structure used for the free energy calculations. "Pose" indicates in which one of the two orientations the ligand is expected to bind given the experimental evidence, where $\mathrm{H}$ is the horizontal pose and $\mathrm{V}$ is the vertical pose. In square brackets are the $95 \%$ confidence intervals of the statistics based on percentile bootstrap.

\section{METHODS}

System Setup. Protein conformations were taken from crystal structures as listed in Tables 1 and 2 (more information on the protein models is in Table S1). If multiple chains were present in the asymmetric unit, only monomer A would be kept for the calculations. Missing atoms in the crystals were modeled with the WHAT-IF web interface ${ }^{27,28}$ and all organic molecules were removed from the system, whereas all crystallographic waters were retained. Proteins were then protonated using the Gromacs tool (Pdb2gmx). The binding poses for RVX-208 and $\mathrm{RVX}-\mathrm{OH}$ were taken from a previous study, ${ }^{10}$ where the poses were identified via docking following the same procedure here used for bromosporine. The preferred binding pose for bromosporine was identified by docking the ligand in an apo X-ray structure of $\mathrm{BRD} 4(1)$ (PDB ID 2OSS) and then performing free energy calculations on the clustered poses. For bromosporine and RVX-208, the highest affinity pose (as predicted by the calculations) was assumed to be conserved and was modeled into the pockets of the other bromodomains by structural alignment to BRD4(1) with PyMOL. For RVX-OH, however, two alternative poses were tested via free energy calculations for all bromodomains binding to this ligand. When placing ligands into the pockets, crystallographic water molecules within $1.4 \AA$ of the organic molecule were removed in order to avoid steric clashes. All other crystallographically resolved water molecules were retained, so that the conserved network of water molecules at the bottom of the cavities was preserved in the calculations. After adding hydrogens with Maestro (v9.5, Schrödinger), the ligands were parametrized with the general AMBER force field ${ }^{29}$ (GAFF v1.7) using AmberTools14. Restrained electrostatic potential (RESP) charges ${ }^{30}\left(\mathrm{HF} / 6-31 \mathrm{G}^{*} / / \mathrm{HF} / 6-31 \mathrm{G}^{*}\right)$ for bromosporine were obtained with the PyRED server ${ }^{31}$ using Gaussian 09 (Rev. D.01). RESP charges for the ligands RVX-208 and RVX-OH were obtained with a geometry optimization followed by a molecular electrostatic potential calculation, both at the $\mathrm{HF} / 6-31 \mathrm{G}^{*}$ level of theory. ESP points were sampled according to the
Merz-Kollman scheme. ${ }^{32,33}$ The RESP fit was done using antechamber in AmberTools14, while all QM calculations were performed in Gaussian 09 (Revision D.01). The PyRED server was not employed in the latter cases due to its unavailability for a number of months in late 2015 . Gromacs topologies and coordinate files were generated from the Amber ones using acpype (v.2014-08-27 Rev. 403). ${ }^{34}$ The Amber99SBILDN force field was used for the protein and the TIP3P model for water molecules. ${ }^{35,36}$ The complexes were solvated in a dodecahedral box with periodic boundary conditions and a minimum distance between the solute and the box of $12 \AA$. Sodium and chloride ions were added to neutralize the systems at the concentration of $0.15 \mathrm{M}$.

Docking. The ligands were docked into an X-ray structure of BRD4(1) (PDB ID 2OSS) using MOE (Chemical Computing Group, v2014.09). All water and organic molecules were removed from the model, with the exception of five water molecules at the bottom of the binding pocket, which are conserved across most bromodomains. The ligands were initially drawn in 2D using Marvin Sketch (ChemAxon, v6.1.0) and a stochastic conformational search was performed in MOE for the generation of multiple 3D conformers. The number of conformers generated was limited to one hundred and duplicates ones (RMSD $<0.25 \AA$ ) were removed. Protein and ligands were parametrized using the AMBER10:EHT force field option in MOE. Thus, the protein parameters were taken from Amber ff99SB, ${ }^{40}$ while the ligand bonded parameters were obtained with 2D Extended Hückel Theory, ${ }^{41}$ the VdW parameters were derived from GAFF, ${ }^{29}$ and the charges from Bond Charge Increments. ${ }^{42}$ Pharmacophores were used for the placement of the ligand during docking. The pharmacophore query was built based on the structure of bound acetyl-lysine, as found in a holo X-ray structure (PDB ID 3UVW), and consisted of a hydrogen-bond acceptor site (mimicking the acetyl oxygen) and a nonpolar site (mimicking the methyl moiety). The London $\Delta G$ function was used for the initial scoring of the poses, and each binding pose was then minimized and rescored with the GBVI/WSA $\Delta G$ function. Duplicate poses were 
Table 2. Summary of the Calculation Results for Bromosporine, along with Information about the Experimental Structural and Affinity Data ${ }^{a}$

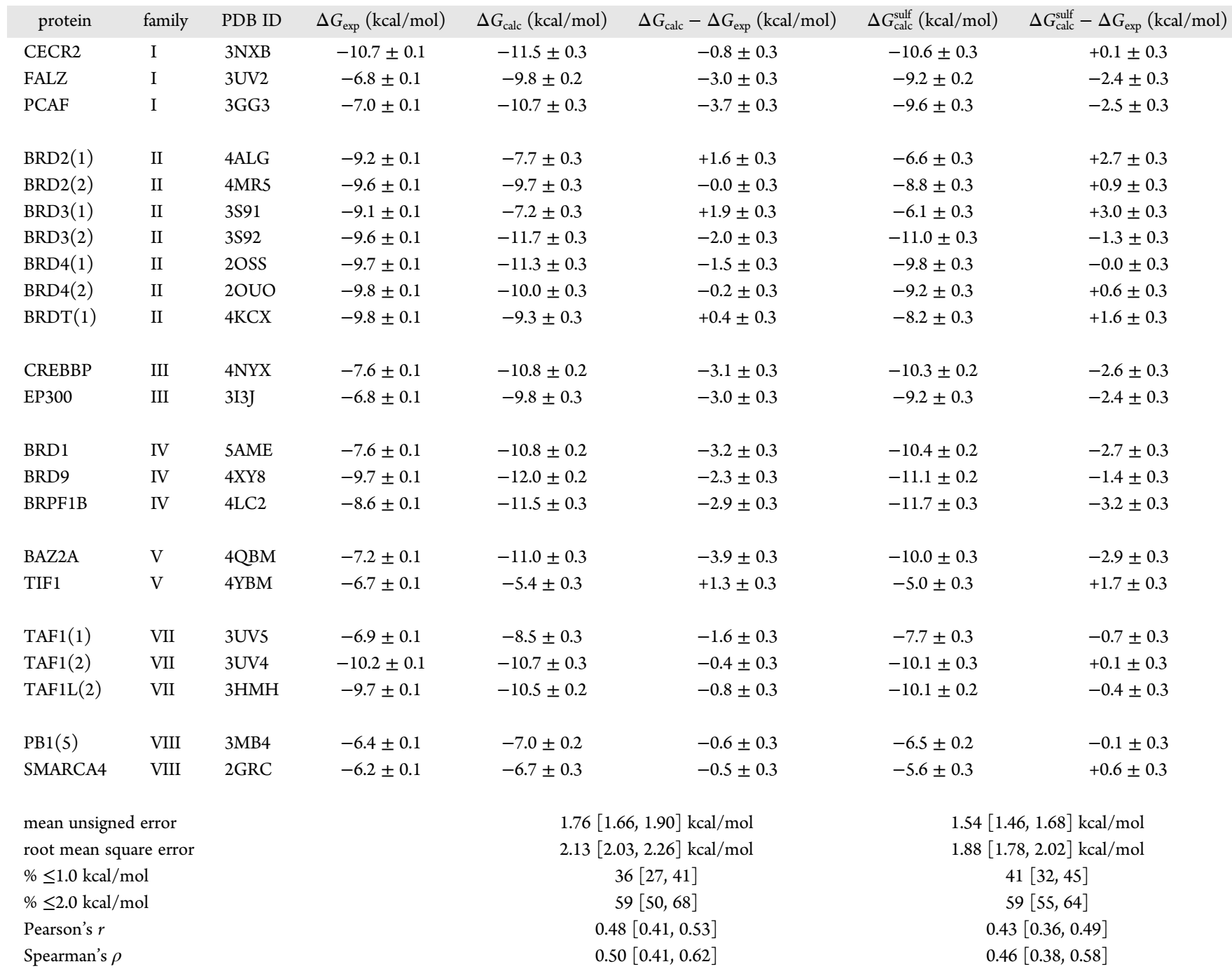

${ }^{a}$ ITC data taken from Picaud et al. ${ }^{39}$ All uncertainties are one standard error of the mean. ITC uncertainties are an estimate based on the standard deviation of the $\Delta G$ values observed in the ABRF-MIRG'02 inter-laboratory assessment. ${ }^{38} \Delta G_{\text {calc }}$ refers to the calculated free energy values with the initial bromosporine model; $\Delta G_{\text {calc }}$ sulf refers to the model with modified torsional parameters for the benzensulfonamide group. Values for the difference $\Delta G_{\text {calc }}-\Delta G_{\text {exp }}$ may appear inconsistent due to rounding. In square brackets are the $95 \%$ confidence intervals of the statistics based on percentile bootstrap.

removed based on their hydrogen-bond and hydrophobic patterns using this option in MOE. In addition, also poses with positive binding free energy as predicted by the GBVI/WSA $\Delta G$ scoring function were removed. The remaining poses were clustered by RMSD with a $3.0 \AA$ cutoff in order to reduce the number of poses to test via free energy calculations, while also retaining a diverse set of binding orientations; in fact, similar binding orientations might interconvert during the simulations, resulting in almost equivalent ensembles. Finally, the best scoring poses within each cluster were selected for free energy calculations. This is the same protocol adopted in our previous study. ${ }^{10}$

Derivation of Small-Molecule Torsional Parameters. The parameters for the two different biaryl torsions present in bromosporine and the two RVX ligands were optimized by least-squares fitting to a $\mathrm{QM}$ torsion scan at the MP2/6-31G* level of theory (Gaussian 09; Rev. D.01) performed every 5 degrees (Figure S1). Three torsion angles present in the benzensulfonamide moiety were also later reparametrized specifically for bromosporine using the program paramfit in AmberTools $14{ }^{43}$ Parameters were derived by fitting the MM energies to single-point energy calculations at the MP2/6-31G* level of theory (Figure S2) performed with Gaussian 09 (Rev. A.02). The parameter search was performed using the hybrid genetic algorithm with a mutation rate of 0.1 ; all other input parameters were used with their default values. A total of 514 conformations were generated by systematic variation of the dihedral angles for the fitting of 3 torsions (4 terms per torsion) within the benzensulfonamide moiety. Conformations with $\mathrm{QM}$ relative energies below $5 \mathrm{kcal} / \mathrm{mol}$ were assigned weights of 1.0 , conformations with energies between 5 and $10 \mathrm{kcal} / \mathrm{mol}$ were assigned weights of 0.5 , and conformations with energies above $10 \mathrm{kcal} / \mathrm{mol}$ were assigned weights of zero. Only the force constant and the phase of the torsion were fit, while the periodicity was fixed. The parameters for all these terms are shown in Figure S2.

Preliminary Molecular Dynamics. In order to improve the fit of the ligands into the binding pockets of the structures used for the free energy calculations, a brief preliminary MD simulation was run for each complex. All simulations were carried out in Gromacs 5.0.4 or Gromacs 5.1. ${ }^{44} 10000$ energy minimization steps were performed using a steepest descent algorithm (the protein-ligand structures obtained after this step were submitted to the CSM-Lig server for scoring). ${ }^{45}$ The system was subsequently simulated for $0.5 \mathrm{~ns}$ in the canonical ensemble and for $1 \mathrm{~ns}$ in the isothermal-isobaric ensemble with harmonic position restraints applied to all solute heavy atoms with a force constant of $1000 \mathrm{~kJ} \mathrm{~mol}^{-1} \mathrm{~nm}^{-2}$. Temperature was coupled using Langevin dynamics 
at the target temperature of $298.15 \mathrm{~K}$, while pressure was coupled using the Berendsen weak coupling algorithm with a target pressure of $1 \mathrm{~atm}^{46-48}$ A $15 \mathrm{~ns}$ unrestrained run was then performed in the NPT ensemble with the Parrinello-Rahman pressure coupling algorithm. ${ }^{49}$ The last frame of the run provided the starting system conformation for the free energy calculations. The last $5 \mathrm{~ns}$ of the unrestrained simulations were also used to obtain equilibrium values for the protein-ligand restraints used during the free energy cycle as described in the next paragraph.

Free Energy Calculations. All calculations were carried out in Gromacs 5.0.4 ${ }^{44}$ (all input files for the ABFE calculations are available as Supporting Information). The ligand van der Waals interactions were decoupled and the charges annihilated using a linear alchemical pathway with $\Delta \lambda=0.05$ for the van der Waals and $\Delta \lambda=0.1$ for the Coulombic transformations. For the addition of the ligand restraints, 12 nonuniformly distributed $\lambda$ values were used $(0.0,0.01,0.025,0.05$, $0.075,0.1,0.15,0.2,0.3,0.5,0.75,1.0)$. A total of 42 windows for the complex simulations and 31 windows for the ligand simulations were therefore employed. For each window, 10000 energy minimization steps were carried out using a steepest descent algorithm. The system was subsequently simulated for $0.5 \mathrm{~ns}$ in the canonical ensemble with harmonic position restraints applied to the solute heavy atoms with a force constant of $1000 \mathrm{~kJ} \mathrm{~mol}^{-1} \mathrm{~nm}^{-2}$. Temperature was coupled using Langevin dynamics with $298.15 \mathrm{~K}$ as the reference temperature. ${ }^{47,48}$ A $1 \mathrm{~ns}$ position restrained run in the isothermal-isobaric ensemble was then performed using the Berendsen weak coupling algorithm and with a target pressure of 1 atm. ${ }^{46}$ Several 15 ns unrestrained production runs were performed for data collection using Hamiltonian-exchange Langevin dynamics in the NPT ensemble with the Parrinello-Rahman pressure coupling scheme. ${ }^{49} \mathrm{~A}$ total of 3 million swaps between any state pair were attempted every 1000 time steps, following the Gibbs sampling scheme proposed by Chodera and Shirts. ${ }^{50}$ The relative position and orientation of the bound ligand with respect to the protein was restrained by means of one distance, two angles, and three dihedral harmonic potentials with force constant of $10 \mathrm{kcal} \mathrm{mol}^{-1} \AA^{-2}\left[\mathrm{rad}^{-2}\right]$. The equilibrium distance and angles for the restraints were taken from the average values of the last $5 \mathrm{~ns}$ of the $15 \mathrm{~ns}$ preliminary MD simulations. The analytical part of the contribution of this set of restraints to the free energy can be evaluated as described by Boresch et al. ${ }^{51}$ The equation used to evaluate this contribution also includes a correction for the standard-state dependence of the binding free energy. ${ }^{51} \mathrm{~A}$ soft-core potential was employed for the van der Waals interactions transformed. ${ }^{52}$ In all simulations, the particle mesh Ewald (PME) algorithm ${ }^{53}$ was used for electrostatic interactions with a real space cutoff of $12 \AA$, a spline order of 6 , a relative tolerance of $10^{-6}$, and a Fourier spacing of 1.0 $\AA$. The Verlet cutoff scheme was used with a van der Waals interaction cutoff of $12 \AA$ and a buffer tolerance of $0.005 \mathrm{~kJ} \mathrm{~mol}^{-1} \mathrm{ps}^{-1}{ }^{54}$ The P-LINCS constraint algorithm was used only on H-bonds. ${ }^{55}$ The Gromacs long-range dispersion correction for energy and pressure was used, and an additional numerical long-range dispersion correction (EXP-LR) was applied as described by Shirts et al. ${ }^{56}$ to address the failure of the isotropic assumption of the analytical correction during simulations of protein-ligand complexes. No correction for box-size effects was applied as it has been shown that, contrary to charged ligands, such effects are negligible for neutral solutes. ${ }^{57}$

Relative free energy calculations were performed in order to estimate the effect of the sulfonamide torsional parameters on the calculated binding free energies for bromosporine. The force constant and phase of the dihedrals were interpolated between the two models using 12 uniformly distributed $\lambda$ values. The equilibration and production phases were carried out as per the protocol described above for absolute free energy calculations. No restraints have been used in this calculations, and all ligand atoms were interacting with the environment during the transformations. For the ligand in solution, five repeats were carried out, while for the protein-ligand complexes simulations a single repeat was performed.

Data Analysis. Free energy estimates were obtained with the implementation of the multiple Bennet acceptance ratio (MBAR) provided with the python package pymbar (https://github.com/choderalab/ pymbar) and using the alchemical analysis tool (https://github.com/ MobleyLab/alchemical-analysis). ${ }^{56,58}$ The data from each lambda state were subsampled in order to include only uncorrelated data-points by calculating the autocorrelation time and statistical inefficiency of the derivative of the potential energy with respect to lambda ( $d h d l)$. Only the dhdl components that were changing at a particular lambda state were used as the observable for the calculation of the autocorrelation times. ${ }^{59}$ The equilibrated region was determined by visualizing the forward and reverse convergence plots as suggested by Klimovich et al. ${ }^{58}$ Multiple such plots were generated by discarding data at increments of $1 \mathrm{~ns}$, from a minimum of $1 \mathrm{~ns}$ to a maximum of $12 \mathrm{~ns}$, and identifying the equilibration time producing a convergence plot with the least drift in free energy in any one direction. The uncertainty of the free energy estimate was taken from the MBAR asymptotic variance-derived uncertainty as the square root of the statistical variance of the total free energy. For the free energy of decoupling the ligand from solution, the mean and sample standard deviation of multiple repeats were used. For bromosporine and RVX-OH, the five initial conformations of the ligand in solution were determined by the five docked poses; for RVX-208, which only had two poses returned by the docking and clustering procedure, ${ }^{10}$ another three snapshots were extracted from the simulations in order to provide three additional random starting conformations. The final binding free energy is the difference between the decoupling of the ligand from the solution and from the solvated complex; the final uncertainty in the binding free energies is thus the root sum square of the uncertainties of ligand and complex calculations. The binding free energies for the bromosporine models with modified torsional parameters were obtained by adding the relative free energy values to the absolute free energy ones that were predicted with the original model. The final uncertainty was derived as the root sum square of the standard errors of the absolute and relative free energies. The $95 \%$ confidence intervals (CIs) for the performance statistics (mean unsigned error (MUE), root-mean-square error (RMSE), Pearson product-moment correlation $\left(r_{\mathrm{p}}\right)$, Spearman rank correlation $\left.\left(r_{\mathrm{s}}\right)\right)$ were calculated with percentile bootstrap, ${ }^{60}$ after building $10^{5}$ bootstrap samples by resampling the experimental and calculated free energies according to their mean and standard errors, assuming normality. Throughout the text we report the $95 \%$ CI in square brackets immediately after the statistics.

\section{RESULTS}

Predicting the Different Poses and Selectivity of Similar Ligands. RVX-208 is an orally available small molecule that is currently being investigated in clinical trials for the treatment of atherosclerosis, diabetes, and cardiovascular diseases. It has been found to inhibit the bromo and extraterminal (BET) family of bromodomains. ${ }^{37,61,62}$ RVX-208 shows a slight selectivity (from about 8- to 21-fold in affinity) for the second (BD2) versus the first bromodomains (BD1) of the BET family. ${ }^{37} \mathrm{RVX}-\mathrm{OH}$ is the synthetic precursor of RVX-208 and also targets the BET bromodomain family; however, it does not show the same preference for the second bromodomains. The compound in fact rearranges in the binding pockets of the first BET bromodomains and binds with a different pose as compared to RVX-208, where it occupies the WP/ZA shelf (see Figure $1 \mathrm{~b}$ for the bromodomain structure). In such a way, RVX-OH achieves similar affinities for both first and second BET bromodomains, abrogating the subtle selectivity observed for RVX-208. The crystallographically observed binding poses for RVX-208 and RVX-OH in the binding pockets of a first and second BET bromodomains are shown in Figure 2. For ease of description, and consistently with the orientation of the figures in this text, we denote the binding orientation of RVX-OH in BD2 pockets as the "vertical" V-pose, and the binding orientation of RVX-OH in BD1 pockets as the "horizontal" H-pose, despite such naming not having any physical or biological meaning. Note that it is not possible for RVX-208 to bind in the H-pose, due to the presence of an additional hydroxyethyl group that would clash with the protein. 

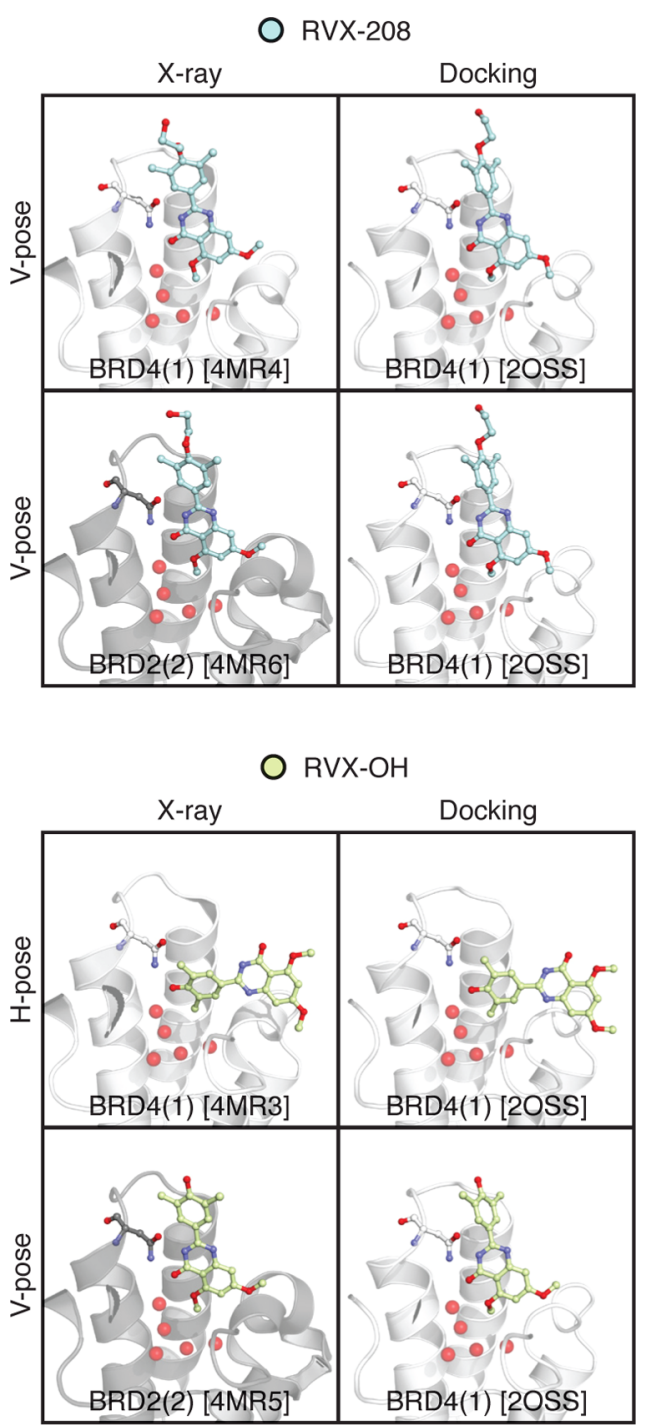

Figure 2. Comparison of the X-ray structures of RVX-208 and RVX-OH in the first (BD1) and second (BD2) BET bromodomains binding pockets, and the corresponding docked structures. RVX-208 binds with the same pose to both $\mathrm{BD} 1$ and $\mathrm{BD} 2$, while RVX-OH adopts two distinct binding orientations. The two ligands have been docked into an apo structure of BRD4(1) (PDB ID 2OSS), and the docking poses best representing the crystal structures are shown. These poses are taken from previous work. ${ }^{10} \mathrm{BD} 1 \mathrm{~s}$ are represented by white cartoons, and $\mathrm{BD} 2 \mathrm{~s}$ by gray cartoons; RVX-208 is highlighted in light blue, and $\mathrm{RVX}-\mathrm{OH}$ in light green; the conserved Asn residue and network of waters are shown as reference points.

These ligands thus represent an interesting case scenario to test the ability of the calculations to identify both the binding modes and slight selectivity of a ligand for multiple proteins. In this study, the 7 BET BRDs (out of 8) with at least a crystal structure available in the RCSB Protein Data Bank (PDB) and affinity data determined by ITC were considered.

The docking pose for RVX-208 was taken from a previous study, in which absolute free energy calculations were shown to be able to identify the crystallographic pose for this ligand in BRD4(1). ${ }^{10}$ Similarly, the two docking poses for RVX-OH corresponding to the $\mathrm{V}$ - and $\mathrm{H}$-poses were taken from the same study; in this case, too, it was shown how the calculations identified the correct pose for BRD4(1). Here, however, we were interested in assessing whether the calculations could predict the difference in its binding orientation between BD1s and BD2s; therefore, we rescored both the $\mathrm{V}$-pose and $\mathrm{H}$-pose for all 7 BRDs considered. The relevant docking poses are shown in Figure 2. The two poses for RVX-OH, and the one for RVX-208, were modeled into the structures of the other six BET bromodomains considered. In order to allow the binding pockets to relax around the ligands, a short (15 ns) MD simulation of the complexes was run before performing the free energy calculations.

Given the two potential binding poses of RVX-OH for the $\mathrm{BET}$ bromodomains, the calculations were run starting from both orientations for all the seven BET bromodomains considered (no structure is yet available for $\operatorname{BRDT}(2)$ ) in order to test whether the computational protocol could reliably identify the correct pose for the first (H-pose) versus second (V-pose) BET bromodomains. Table 1 summarizes the results of the calculations obtained for RVX-OH, and shows how the orientation that would be expected to be the more stable based on the crystallographic evidence was always predicted to have a more negative binding free energy value, i.e. a higher affinity, than the other competing orientation. For each bromodomain/RVX-OH complex we then took the higher affinity pose as being the most stable orientation, and compared the corresponding binding free energy value to the ITC data. As previously mentioned, RVX-208 can bind only in one orientation ( $\mathrm{V}$-pose), thus this exercise was not carried out for this ligand. However, it needs to be kept in mind that it is the retrospective nature of this study that has allowed us to confidently make such an assumption. In a prospective drug discovery scenario, it is likely that limited information will be available on the compound being investigated, so that one might need to be more careful when assuming a conserved pose across different proteins.

Table 1 and Figure 3 summarize the correspondence of the computational predictions to the ITC data for the two ligands together (full breakdown of free energy terms in Table S2). It is possible to see how, in this case, the predictions reproduced experimental values in absolute terms extremely well. All of the predicted binding free energies were within $2 \mathrm{kcal} / \mathrm{mol}$ of the ITC values, and about two-thirds were within $1 \mathrm{kcal} / \mathrm{mol}$. This resulted in low MUE and RMSE: $0.81[0.74,0.90] \mathrm{kcal} / \mathrm{mol}$ and $0.95[0.87,1.05] \mathrm{kcal} / \mathrm{mol}$, respectively. The correlation with ITC values was particularly good too for absolute calculations, with a Pearson correlation $\left(r_{\mathrm{p}}\right)$ of $0.75[0.67,0.80]$ and a Spearman $\left(r_{s}\right)$ of 0.78 [0.64, 0.85]. Considering RVX-208 alone, the calculations achieved $r_{\mathrm{p}}=0.84[0.76,0.90]$ and $r_{\mathrm{s}}=0.75$ $[0.71,0.93]$. On the other hand, when considering RVX-OH only, lower correlation values with larger uncertainties were returned $\left(r_{\mathrm{p}}=0.49[0.27,0.66]\right.$ and $\left.r_{\mathrm{s}}=0.46[0.14,0.71]\right)$ despite the calculations performing well in terms of absolute errors for both ligands. This is expected given the small dynamic range of $1.3 \mathrm{kcal} / \mathrm{mol}$ when considering RVX-OH alone.

If the predictions were grouped into first $[\mathrm{BRD} 2(1), \mathrm{BRD} 3(1)$, $\operatorname{BRD} 4(1), \operatorname{BRDT}(1)]$ and second [BRD2(2), BRD3(2), BRD4(2)] BET bromodomains, it would be possible to anticipate the slight selectivity of RVX-208 for BD2s, as one can visually gather from Figure 3. In fact, the sample mean and standard deviation of RVX-208 binding free energies for BD1s were of $-6.5 \pm 0.7 \mathrm{kcal} / \mathrm{mol}$, while for BD2s of $-8.6 \pm 1.1 \mathrm{kcal} / \mathrm{mol}$. A two-tailed $t$ test returns a $p$-value of 0.03 , which would suggest that the predicted difference in affinity between the two groups is unlikely to be caused by chance. Contrariwise, RVX-OH was predicted to have an average binding free energy of $-8.5 \pm$ $1.6 \mathrm{kcal} / \mathrm{mol}$ for $\mathrm{BD} 1 \mathrm{~s}$ and of $-7.9 \pm 0.6 \mathrm{kcal} / \mathrm{mol}$ for $\mathrm{BD} 2 \mathrm{~s}$, 

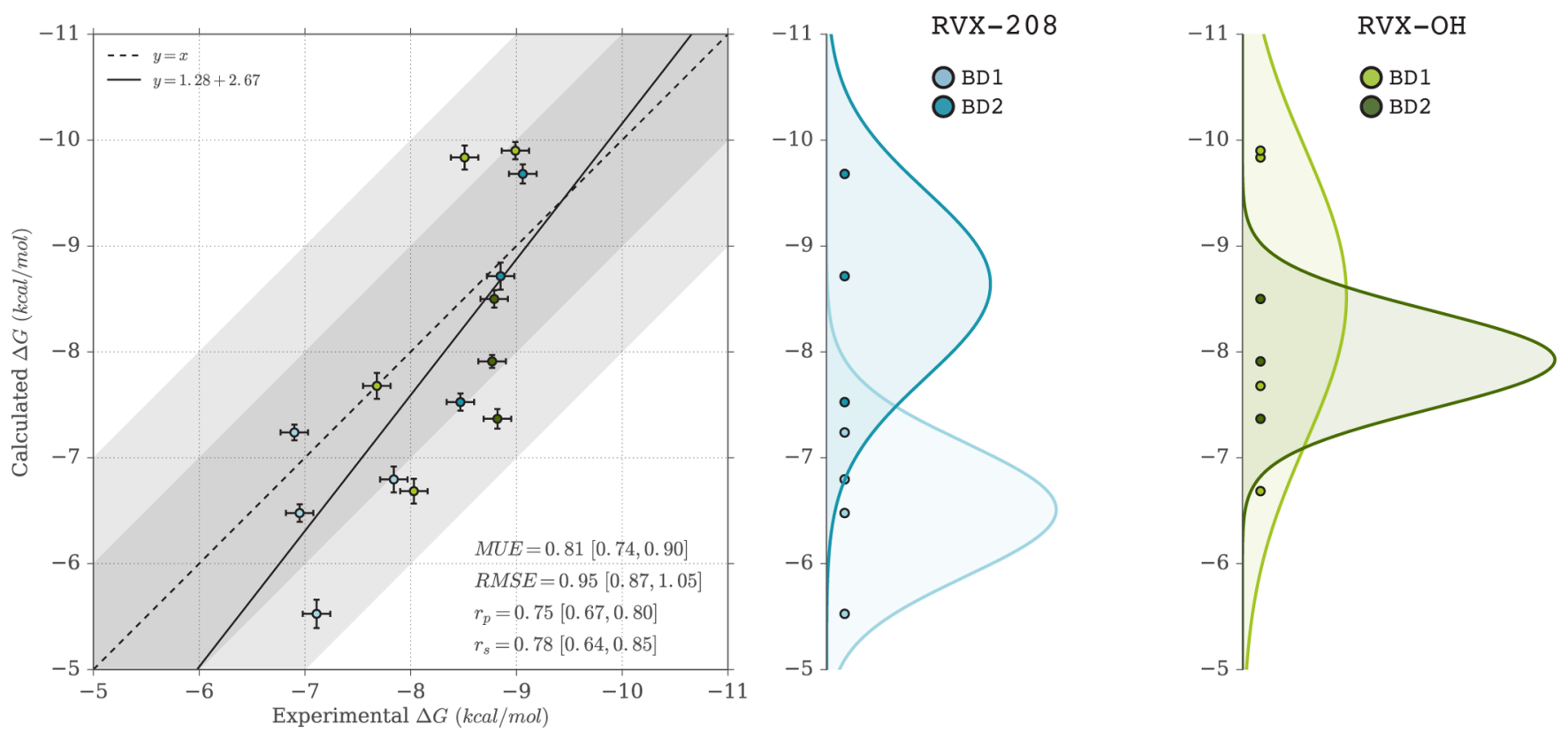

Figure 3. Scatter plot of calculated versus experimental affinities for the compounds RVX-208 and RVX-OH. The shaded gray areas indicate where the 1 and $2 \mathrm{kcal} / \mathrm{mol}$ error boundaries lie. On the right-hand side are the distributions of the calculated binding free energies for the two ligands, binding to $\mathrm{BD} 1 \mathrm{~s}$ (light blue and light green) and BD2s (dark blue and dark green); Gaussian curves have been fitted to the data.

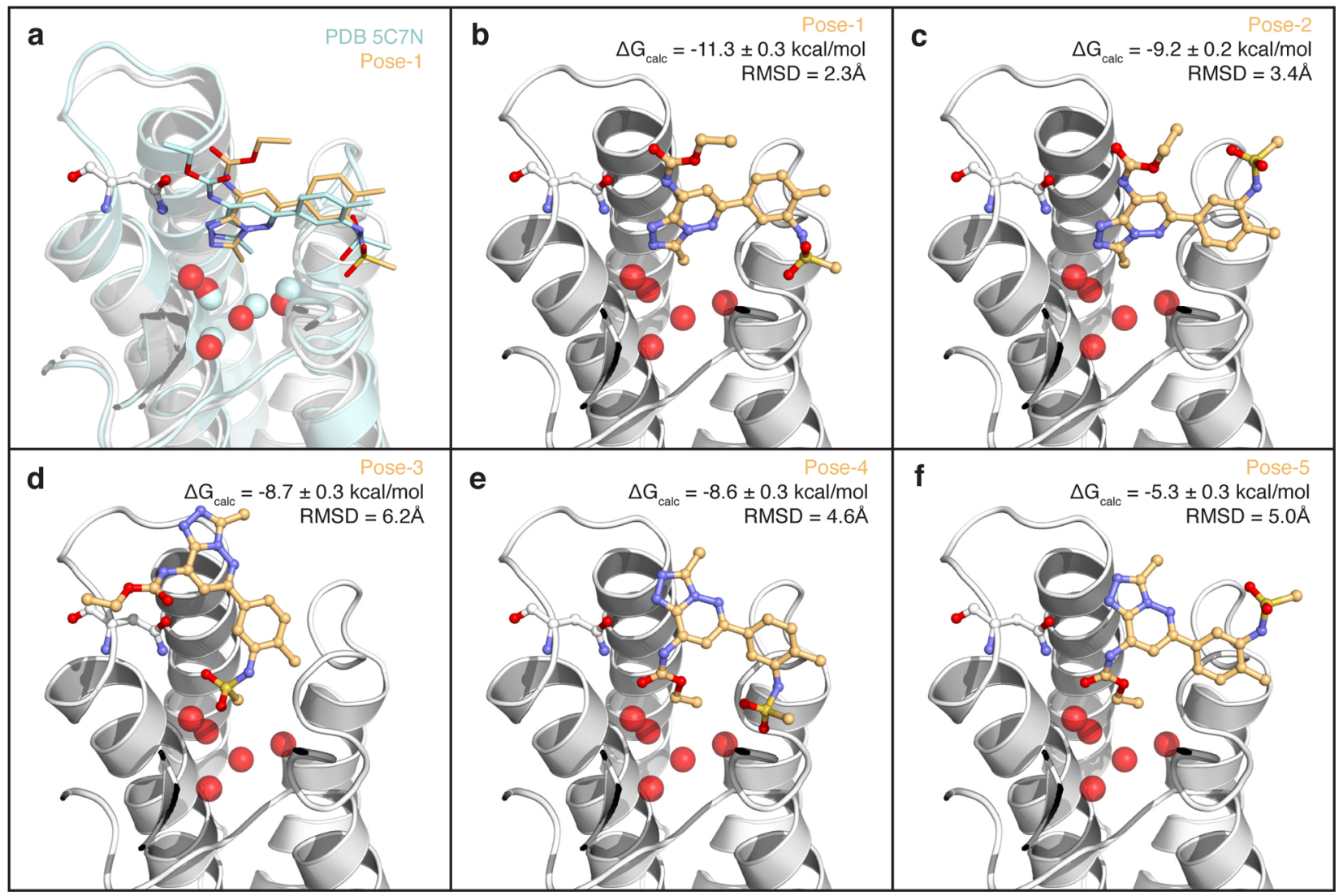

Figure 4. Docking poses for bromosporine in the binding pocket of BRD4(1). (a) Comparison of the pose with highest predicted affinity to the X-ray structure of bromosporine in complex with BRPF1B (PDB-ID 5C7N). (b-e) The five diverse bromosporine poses suggested by docking, with their predicted binding affinity and RMSD with respect to the binding mode found in the crystal structure.

showing a larger overlap of their distributions and consequently returning a $p$-value of 0.57 when applying a two-tailed $t$ test, correctly suggesting the probable lack of any preference for either the first or second BET bromodomains.
For comparison, we used a recent machine learning scoring function to test whether similar results might have been achievable with a much cheaper computational method. More specifically, we used CSM-Lig, a machine-learning method that relies 
a

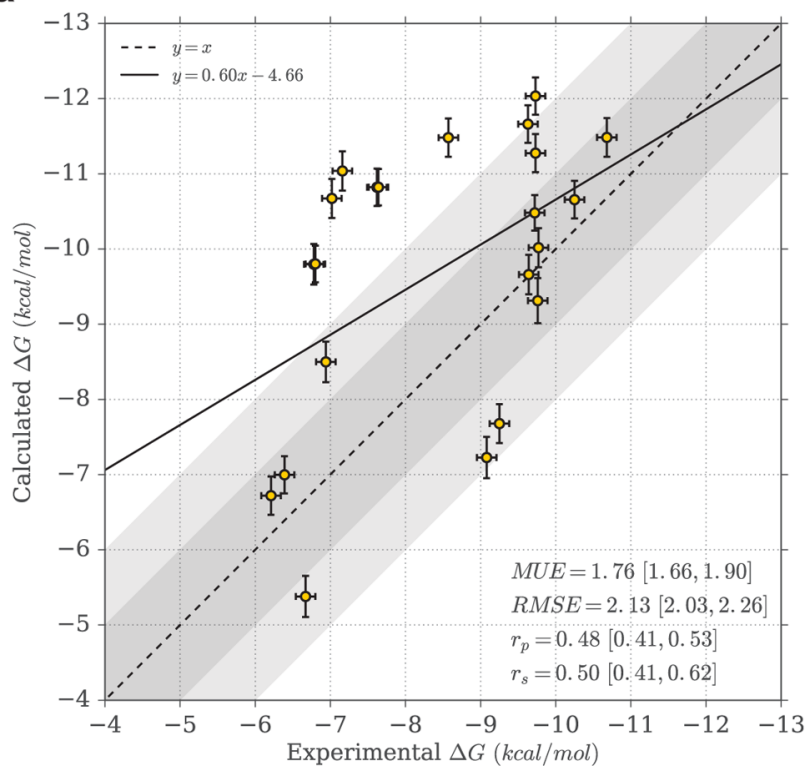

b

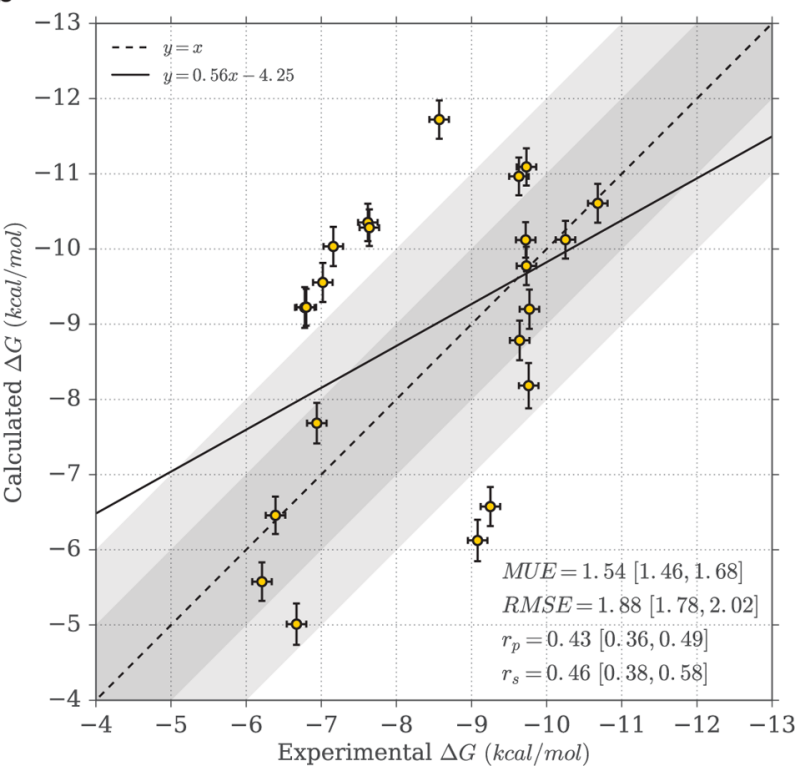

Figure 5. Scatter plots of calculated versus experimental binding free energies for bromosporine. (a) Calculated binding free energies for the initial bromosporine model. (b) Calculated binding free energies for the bromosporine model with optimized benzensulfonamide torsions. The shaded gray areas indicate where the 1 and $2 \mathrm{kcal} / \mathrm{mol}$ error boundaries lie.

on graph-based signatures and showed a performance statistically comparable to RF-Score ${ }^{63,64}$ and superior to 18 other common scoring functions. ${ }^{45}$ The input protein-ligand structures were the docking poses modeled in all BRDs considered after minimization of the whole system in explicit solvent using Gromacs. The scoring function identified the correct pose for $\mathrm{RVX}-\mathrm{OH}$ five out of seven times, and returned more modest correlation with experimental affinities $\left(r_{\mathrm{p}}=0.44\right.$ and $\left.r_{\mathrm{s}}=0.35\right)$. Furthermore, the predictions systematically underestimated the binding for these two ligands (Table S5), returning large values for the MUE and RMSE of 5.82 and $5.86 \mathrm{kcal} / \mathrm{mol}$, respectively. No prediction was within $2 \mathrm{kcal} / \mathrm{mol}$ of the experimental value. Figure S3 plots the predicted versus experimental binding free energies and shows how the scoring-based predictions deviated substantially from the line of identity.

Reproducing the Affinity Profile of a Broad-Spectrum Inhibitor. Bromosporine (Figure 1c) is a broad-spectrum bromodomain inhibitor that binds across all bromodomain families with a relatively wide range of affinities (from $\sim 25 \mu \mathrm{M}$ to $\sim$ $10 \mathrm{nM}$; from -6.2 to $-10.7 \mathrm{kcal} / \mathrm{mol}$ in binding free energy terms). As affinities for a number of BRDs have been experimentally determined by ITC (Table 2), ${ }^{39}$ it is an ideal system to test the ability of free energy calculations to capture the affinity profile of a ligand across many similar binding pockets. As for RVX-OH and RVX-208, all bromodomains with at least a crystal structure available in the PDB and affinity data determined by ITC were considered, for a total of 22 bromosporine/BRD pairs.

At the outset of this study, no structure of bromosporine in complex with a bromodomain had been deposited in the PDB. We therefore docked the ligand in the binding pocket of BRD4(1) in order to determine its likely binding pose. Five different clusters of poses were identified and free energy calculations were performed on one pose from each cluster in order to determine the most stable orientation. These were distinct poses that did not interconvert within the simulations time scale. Figure 4 provides an overview of these docking poses, ranked by their predicted affinity. In July 2015 the first bromosporine structure in complex with a human bromodomain (BRPF1B, family IV) was released on the PDB (PDB ID 5C7N), ${ }^{65}$ so that we were able to compare the docked poses to this experimentally resolved structure. Figure $4 \mathrm{a}$ shows the superimposition of the docking pose with highest predicted affinity in BRD4(1) and the $\mathrm{X}$-ray structure of bromosporine in complex with BRPF1B. The predicted pose resembled well the crystallographic orientation, with a RMSD of $2.3 \AA$, mainly due to a rotation of the ethyl carbamate side chain. The fact that bromosporine was shown to bind to BRPF1B in the same fashion as predicted for BRD4(1) also gave us confidence that the binding mode for this ligand was conserved across bromodomain families. The second pose (Figure 4c) was close to the experimental structure as well (RMSD of $3.4 \AA$ ), but with the sulfonamide group directed toward the solvent rather than making contacts with the protein. The remaining three poses (Figure $4 \mathrm{~d}-\mathrm{f}$ ) were bound in substantially different orientations as compared to the first two docking poses and the crystallographic pose, as indicated by the larger RMSDs of 4.6, 5.0, and $6.2 \AA$. The binding affinity of bromosporine for BRD4(1) was, however, overestimated by over $1 \mathrm{kcal} / \mathrm{mol}$ : the calculated free energy of the most stable pose was predicted to be $-11.3 \pm 0.3 \mathrm{kcal} / \mathrm{mol}$, while the ITC measure is of $-9.7 \pm 0.1 \mathrm{kcal} / \mathrm{mol}$. Correspondingly, the docking poses $\mathrm{c}, \mathrm{d}$, and e are unexpectedly assigned large affinities while binding very differently from the X-ray pose.

After establishing the most likely binding orientation of bromosporine in BRD pockets via docking, the ligand was modeled into the X-ray structures of the other 21 bromodomains, from 7 of the 8 bromodomain families. As for the RVX ligands, a short MD simulation of the complexes was run before performing free energy calculations for all the bromosporine/ BRD pairs. The results of the calculations are summarized in Table 2 (full breakdown of free energy terms in Table S3) and Figure 5a. A reasonable overall agreement with experiment in terms of absolute values was achieved, with MUE of 1.76 $[1.66,1.90] \mathrm{kcal} / \mathrm{mol}$ and RMSE of $2.13[2.03,2.26] \mathrm{kcal} / \mathrm{mol}$. Roughly a third of the results were within $1 \mathrm{kcal} / \mathrm{mol}$ of the ITC 
values, and two-thirds within $2 \mathrm{kcal} / \mathrm{mol}$. However, this left another third of the results being off by at least $2 \mathrm{kcal} / \mathrm{mol}$, which corresponds to about a 30 -fold error in the dissociation constant. The correlation with experiment was reasonable too, but not as strong as for RVX-OH/RVX-208, with $r_{\mathrm{p}}=0.48$ [0.41, 0.53] and $r_{\mathrm{s}}=0.50[0.41,0.62]$. The low number of samples from each bromodomain family hampered a meaningful statistical analysis, and in fact a one-way analysis of variance revealed that the error distribution of each subfamily did not differ in a statistically significant manner from any other subfamily. However, there seemed to be a pattern where calculations involving bromodomains from subfamilies II, VII, and VIII, returned better results than other subfamilies.

Overall, in this set of predictions, the binding free energies are overestimated; in fact, the mean signed error was of -1.29 $[-1.42,-1.17] \mathrm{kcal} / \mathrm{mol}$. As we were interested in identifying possible sources for this issue, we evaluated the effect of the charge model on the results by carrying out relative calculations where RESP charges were transformed into AM1-BCC ones (data not shown). However, this change aggravated the overestimation issue, resulting in higher MUE and RMSE (2.15 and $2.54 \mathrm{kcal} / \mathrm{mol}$, respectively) and a more negative mean signed error $(-2.00 \mathrm{kcal} / \mathrm{mol})$, while leaving the correlations almost unvaried $\left(r_{\mathrm{p}}=0.46\right.$ and $\left.r_{\mathrm{s}}=0.48\right)$. We then focused on the parameters of the soft dihedrals present in the molecule, since such terms are known to have limited transferability across different molecules and might lead to inaccurate sampling of ligand conformations. ${ }^{66}$ In particular, chemical groups such as sulfonamides are especially challenging when considering that also quantum effects like the interaction of the nitrogen lone pair with antibonding orbitals involving the sulfur affect the torsional energy around the $\mathrm{N}-\mathrm{S}$ bond. ${ }^{67,68}$ For these reasons, we decided to optimize the torsional parameters that determine the ensemble of conformations explored by the benzensulfonamide moiety in bromosporine. These dihedral terms were optimized specifically for bromosporine using the program paramfit ${ }^{43}$ where the target energies were obtained with single-point energy calculations at the MP2/6-31G* level of theory. The parameters derived in such a way are specific for the bromosporine model we used, and provided a better set of parameters in terms of agreement with QM energies in vacuo (Figure S2). In order to estimate the effect of the new set of torsional parameters on the affinity predictions, RBFE calculations were carried out. The predicted binding free energies for the optimized model were derived by adding the $\Delta \Delta G$ obtained from the RBFE calculations, to the $\Delta G_{\text {calc }}$ values obtained with ABFE calculations for the initial model. Table 2 and Figure $5 b$ summarize the results obtained for the bromosporine models with modified torsional parameters for the benzensulfonamide group (full breakdown of the free energy terms in Table S4). It is possible to note how the optimization of the sulfonamide moiety had a positive effect on the absolute errors of the calculations, with MUE decreasing to 1.54 $[1.46,1.68] \mathrm{kcal} / \mathrm{mol}$ and RMSE to $1.88[1.78,2.02] \mathrm{kcal} / \mathrm{mol}$. The percentage of predicted binding free energy values with error relative to ITC of less than $1 \mathrm{kcal} / \mathrm{mol}$ also increased slightly, while the percentage of less than $2 \mathrm{kcal} / \mathrm{mol}$ remained constant. Importantly, the strong overestimation problem was alleviated, so that the errors were distributed more symmetrically around zero, with a mean signed error of $-0.53[-0.65,-0.41]$ $\mathrm{kcal} / \mathrm{mol}$. On the other hand, unexpectedly, the correlation between calculated and experimental affinities slightly deteriorated $\left(r_{\mathrm{p}}=0.43[0.36,0.49]\right.$ and $\left.r_{\mathrm{s}}=0.46[0.38,0.58]\right)$. We further proceeded to derive optimized parameters for other soft torsions found in bromosporine: the ones found in the ethyl carbamate side chain, and again the one between the two aromatic rings. However, new parameters for these dihedrals did not result in improved agreement with experiment in terms of absolute error or correlation (data not shown). It thus appeared that the sulfonamide parameters only (among the one tested) were contributing toward the systematic overestimation of the binding free energies for bromosporine. Nonetheless, the modification did not completely solve the overestimation issue, and the overall accuracy of the predictions for this ligand was still modest as compared to what achieved with the two RVX ligands, and what previously reported for the binding of 11 other compounds to BRD4(1). ${ }^{10}$ This suggests there probably are other factors contributing toward the errors in the predictions for this test case, which we have not yet been able to identify.

As it was done for RVX-OH and RVX-208, the ABFE calculations were also compared to a computationally cheaper, yet state of the art, scoring function. ${ }^{45}$ In this case too, the CSM-Lig method returned lower correlation to experiment as compared to ABFE, with $r_{\mathrm{p}}=0.22$ and $r_{\mathrm{s}}=0.19$ (Figure S3). Moreover, the predictions deviated substantially from the ITC measurements, returning a MUE of $5.38 \mathrm{kcal} / \mathrm{mol}$ and a RMSE of $5.62 \mathrm{kcal} / \mathrm{mol}$. However, contrary to the case in RVX-OH and RVX-208, for bromosporine this approach overestimated binding, with most binding free energies being around $-14 \mathrm{kcal} / \mathrm{mol}$ (Table S6). As for the RVX compounds, none of these predictions was within $2 \mathrm{kcal} / \mathrm{mol}$ of the experimentally measured binding free energy.

\section{DISCUSSION}

Here, we presented two test cases in which the prediction of the different affinities for single ligands binding to multiple bromodomains was attempted. Excellent agreement with experimental data was achieved for the compounds RVX-OH and RVX-208, while the broad-spectrum inhibitor bromosporine proved to be more challenging. It is important to note how the quality of the ligand parameters might vary largely between different small molecules depending on the chemical groups present. The fact that reparameterization of the soft torsions in the benzensulfonamide moiety for the bromosporine model alleviated the systematic overestimation of binding affinities corroborates the hypothesis that transferability of dihedral parameters in small molecules can be an issue. Parameterization approaches where some of the ligand parameters, such as charges and torsions, are derived on an ad hoc basis for the specific molecule at hand might provide more accurate organic molecule models for the study of dynamics and binding free energies. ${ }^{66}$ Certainly, this is not the only issue with classical force field models; nonetheless, it is one that can be readily tackled without modification of current functional forms. Small-molecule force fields are also relatively young when compared to protein force fields, with much room for improvement. As demonstrated by the recent OPLS3 force field, little adjustments such as off-site charges and careful parametrization that takes advantage of increasing availability of experimental data can provide better models of protein-ligand interactions within the framework of standard functional forms. ${ }^{69}$ In this regard, also work on host-guest systems, for which highly converged calculations can be obtained, will prove valuable for the parametrization of the next generations of small-molecule force fields. ${ }^{70,71}$

For bromosporine, even with the optimization of torsional parameters, reasonable but not excellent agreement with ITC data was eventually obtained, in particular in terms of correlation. This was caused by large $(>2 \mathrm{kcal} / \mathrm{mol})$ overestimation or 
underestimation of the binding free energy in a number of cases. The identification of the causes for such disagreement with ITC proved challenging, as a large number of potential reasons can be conceived. The imprecision of the calculations alone cannot justify the magnitude of such errors (see SI Text T1 for a more detailed analysis of the uncertainty estimates, and SI Text T2 for a more comprehensive discussion on the possible sources of error). However, severe sampling issues, such as the failure to access relevant states (e.g., side-chain rotamers or loop motions), might contribute toward the errors. ${ }^{25,72}$ Identifying specific sampling deficiencies on a case-by-case basis can, however, be both challenging and impractical, in particular with the prospect of being able to apply these calculations to large numbers of protein-ligand pairs. The combination of free energy calculations with enhanced sampling techniques might thus be beneficial, as it does not require the identification of specific degrees of freedom that have been under-sampled. ${ }^{12,73,74}$ Force field inaccuracies can also have a large effect on the calculations. Aside from bonded terms, inaccuracies in the nonbonded parameters can cause over/under-estimation of interaction energies and consequently protein-ligand affinities. While parameters such as the Lennard-Jones can be improved with larger sets of experimental data, ${ }^{75,76}$ effects such as polarization, which can also affect the results of the calculations across different proteins, will likely need a more substantial alteration of the current models. ${ }^{77-79}$ Cation- $\pi$ interactions have been shown to be important for the binding of certain ligands to bromodomains such as CREBBP, ${ }^{80-82}$ and this is an example of a protein-ligand interaction that is unlikely to be captured accurately by current classical force fields. ${ }^{83}$ These are only some of the challenges that might, currently, negatively affect the accuracy, reliability, and generality of binding free energy calculations. Tautomerisation, changes of protonation states, and effects due to the presence of the buffer, are other issues that may arise and can also affect predicted protein-ligand binding free energies. $^{83,84}$

Despite all the challenges, the results presented here are encouraging. In terms of absolute errors, the predictions achieved a RMSE below the $1 \mathrm{kcal} / \mathrm{mol}$ mark for the RVX$\mathrm{OH} / \mathrm{RVX}-208$ test case and below $2 \mathrm{kcal} / \mathrm{mol}$ for bromosporine. The ability to quantitatively reproduce binding free energies, rather than only correlate with them, has been a difficult task for decades and for most computational approaches. ${ }^{10,11}$ Now, physics-based models of chemical and biological matter and computer simulations seem to be able to provide sufficient physical accuracy and degree of sampling in order to reproduce one of the most important thermodynamic quantities in biology and pharmacology. It is, however, wise to exercise some cautious optimism. In fact, while it is important to start assessing the accuracy and precision of the calculations on specific systems, the test sets used so far for ABFE calculations are limited in their size and diversity. As the computer power available continues to increase, along with algorithmic improvements, ${ }^{85}$ in the future it will be possible to expand such validation studies to larger benchmark sets, ${ }^{86}$ similarly to what has been only recently possible for RBFE calculations. ${ }^{12,19,87}$ With an expanded chemical and biological space used for testing, the results will provide a better picture of the capabilities and pitfalls of the methodology as well as its most fruitful fields of applicability.

We also showed how rigorous calculations based on $\mathrm{MD}$, in these two test cases, outperformed a state-of-the-art machine learning scoring function, providing superior agreement to experiment both in terms of correlation and absolute error.
Nonetheless, it is important to realize that the difference in computational cost of the two approaches is vast. While, currently, ABFE calculations need the use computer clusters for hours to days, scoring functions can return a prediction within seconds on a regular desktop machine. The calculations reported here also required considerable human time for their setup. The use of a few ad hoc in-house scripts aided the process; however, ligand parametrization, generation of input files, setup of restraints, and the data analysis all involved some degree of human intervention. The lack of automated workflows might be partly due the method still being explored, so that strong consensus around a generally applicable "standard protocol" is missing. Nonetheless, the human effort needed to familiarize and setup the calculations does constitute an entry barrier for new users and might prevent a widespread use. In fact, the development of automated and user-friendly software has contributed to the spread of RBFE calculations in only the past few years. ${ }^{12,88,89}$ Credit must be given, however, to developers of freely available simulation software who keep improving the capabilities of these programs for free energy calculations. ${ }^{90-92}$ We are confident that, gradually, as interest in these calculations increases thanks to further developments and testing, workflows will become more and more automated and user-friendly. The reader who is already interested in learning how to run these calculations can find some additional considerations, and references to background information, in SI Text T3.

In our opinion, ABFE calculations can have the highest potential at the hit discovery and lead development stages, complementing established techniques such as docking as well as emerging ones like RBFE calculations. The objective is not to have perfect predictions and replace experiments, but to shorten and reduce the cost of development cycles, and to promote the testing of novel and synthetically challenging hypotheses. During hit discovery, one can foresee virtual screening protocols where a number of techniques are employed hierarchically. ${ }^{93}$ Docking will always be faster than ABFE calculations, and could be used for the initial enrichment of binders within the library screened; this could be followed by intermediate approaches in terms of accuracy and speed, such as implicit solvent calculations like Molecular Mechanics Poisson-Boltzmann Surface Area (MMPBSA); ${ }^{94}$ finally, for the most accurate rescoring and ranking, ABFE calculations may be used. At the lead development stage, such calculations could be employed to assess the likelihood of a ligand with a very different scaffold to retain binding, or to assess different ligands for their ability to hit the target selectively, as discussed in this study.

\section{CONCLUSION}

In this work, we have shown how absolute binding free energy calculations based on molecular dynamics can be applied in order to computationally predict the affinity profile of a ligand across multiple proteins with no prior knowledge of the structure of the complexes or affinities for similar ligands. The prediction of binding affinities and the engineering of selectivity are both still major challenges in drug design; however, with increasing computational power at our hands and with more rigorous and general computational approaches, these problems can start being tackled. It is here shown how a thorough simulation protocol can, in the cases considered, achieve good correlation and agreement with experimental values for drug-like ligands. It is, however, evident that the accuracy of the calculations is system dependent, and further investigation is needed in order to assess the effectiveness of such predictions in different case 
scenarios and on different protein and ligand systems. While the accuracy of these calculations for other protein families still needs to be established, the performance of the predictions here reported is encouraging.

\section{ASSOCIATED CONTENT}

\section{S Supporting Information}

The Supporting Information is available free of charge on the ACS Publications website at DOI: 10.1021 /jacs.6b11467.

Details of the proteins, details of reparameterization data, machine learning scoring results, breakdown of free energy components, discussion of the precision of the calculations, and discussion of the potential sources of error and additional considerations useful for new users, including Figures S1-S3 and Tables S1-S6 (PDF)

All input files pertaining to the calculations, provided as a compressed archive file (ZIP)

\section{AUTHOR INFORMATION}

\section{Corresponding Author}

*philip.biggin@bioch.ox.ac.uk

\section{ORCID ${ }^{\circ}$}

Matteo Aldeghi: 0000-0003-0019-8806

Philip C. Biggin: 0000-0001-5100-8836

Notes

The authors declare no competing financial interest.

\section{ACKNOWLEDGMENTS}

M.A. is supported by the EPSRC and Evotec via the Systems Approaches to Biomedical Sciences Doctoral Training Centre (EP/G037280/1). S.K. is supported by the SGC, a registered charity (number 1097737) that receives funds from AbbVie, Bayer, Boehringer Ingelheim, the Canada Foundation for Innovation, the Canadian Institutes for Health Research, Genome Canada, GlaxoSmithKline, Janssen, Lilly Canada, the Novartis Research Foundation, the Ontario Ministry of Economic Development and Innovation, Pfizer, Takeda, and the Wellcome Trust $[092809 / \mathrm{Z} / 10 / \mathrm{Z}]$. We thank the Advanced Research Computing (ARC) facility, the EPSRC UK National Service for Computational Chemistry Software (NSCCS) at Imperial College London (grant no. EP/J003921/1) and the ARCHER UK National Supercomputing Services for computer time granted via the UK High-End Computing Consortium for Biomolecular Simulation, HECBioSim (http://www.hecbiosim.ac.uk), supported by EPSRC (grant no. EP/L000253/1). M.A. would like to thank Davide Sabbadin (Syngenta) for helpful suggestions on small-molecule force field parameters, as well as the participants of the 2016 workshop on Free Energy Methods in Drug Design for useful discussions.

\section{REFERENCES}

(1) Huggins, D. J.; Sherman, W.; Tidor, B. J. Med. Chem. 2012, 55, 1424.

(2) Peters, J.-U. J. Med. Chem. 2013, 56, 8955.

(3) Ciceri, P.; Müller, S.; O’Mahony, A.; Fedorov, O.; Filippakopoulos, P.; Hunt, J. P.; Lasater, E. A.; Pallares, G.; Picaud, S.; Wells, C.; Martin, S.; Wodicka, L. M.; Shah, N. P.; Treiber, D. K.; Knapp, S. Nat. Chem. Biol. 2014, 10, 305.

(4) Uitdehaag, J. C. M.; Verkaar, F.; Alwan, H.; de Man, J.; Buijsman, R. C.; Zaman, G. J. R. Br. J. Pharmacol. 2012, 166, 858.
(5) Davis, M. I.; Hunt, J. P.; Herrgard, S.; Ciceri, P.; Wodicka, L. M.; Pallares, G.; Hocker, M.; Treiber, D. K.; Zarrinkar, P. P. Nat. Biotechnol. 2011, 29, 1046

(6) Filippakopoulos, P.; Knapp, S. Nat. Rev. Drug Discovery 2014, 13, 337.

(7) Filippakopoulos, P.; Picaud, S.; Mangos, M.; Keates, T.; Lambert, J.-P.; Barsyte-Lovejoy, D.; Felletar, I.; Volkmer, R.; Müller, S.; Pawson, T.; Gingras, A.-C.; Arrowsmith, C. H.; Knapp, S. Cell 2012, 149, 214.

(8) Henrich, S.; Feierberg, I.; Wang, T.; Blomberg, N.; Wade, R. Proteins: Struct., Funct., Genet. 2010, 78, 135.

(9) Mobley, D. L.; Dill, K. A. Structure 2009, 17, 489.

(10) Aldeghi, M.; Heifetz, A.; Bodkin, M. J.; Knapp, S.; Biggin, P. C. Chem. Sci. 2016, 7, 207.

(11) Chipot, C. Wiley Interdiscip. Rev: Comp. Mol. Sci. 2014, 4, 71.

(12) Wang, L.; Wu, Y.; Deng, Y.; Kim, B.; Pierce, L.; Krilov, G.; Lupyan, D.; Robinson, S.; Dahlgren, M. K.; Greenwood, J.; Romero, D. L.; Masse, C.; Knight, J. L.; Steinbrecher, T.; Beuming, T.; Damm, W.; Harder, E.; Sherman, W.; Brewer, M.; Wester, R.; Murcko, M.; Frye, L.; Farid, R.; Lin, T.; Mobley, D. L.; Jorgensen, W. L.; Berne, B. J.; Friesner, R. A.; Abel, R. J. Am. Chem. Soc. 2015, 137, 2695.

(13) Christ, C. D.; Fox, T. J. Chem. Inf. Model. 2014, 54, 108.

(14) Mikulskis, P.; Genheden, S.; Ryde, U. J. Chem. Inf. Model. 2014, 54, 2794.

(15) Gapsys, V.; Michielssens, S.; Seeliger, D.; de Groot, B. L. J. Comput. Chem. 2015, 36, 348.

(16) Samsudin, F.; Parker, J. L.; Sansom, M. S. P.; Newstead, S.; Fowler, P. W. Cell Chem. Biol. 2016, 23, 299.

(17) Leonis, G.; Steinbrecher, T.; Papadopoulos, M. G. J. Chem. Inf. Model. 2013, 53, 2141.

(18) Michielssens, S.; Peters, J. H.; Ban, D.; Pratihar, S.; Seeliger, D.; Sharma, M.; Giller, K.; Sabo, T. M.; Becker, S.; Lee, D.; Griesinger, C.; de Groot, B. L. Angew. Chem., Int. Ed. 2014, 53, 10367.

(19) Gapsys, V.; Michielssens, S.; Seeliger, D.; de Groot, B. L. Angew. Chem., Int. Ed. 2016, 55, 7364.

(20) Lin, Y.-L.; Meng, Y.; Huang, L.; Roux, B. J. Am. Chem. Soc. 2014, 136, 14753.

(21) Lin, Y.-L.; Meng, Y.; Jiang, W.; Roux, B. Proc. Natl. Acad. Sci. U. S. A. 2013, 110, 1664.

(22) Lin, Y.-L.; Roux, B. J. Am. Chem. Soc. 2013, 135, 14741.

(23) Boyce, S. E.; Mobley, D. L.; Rocklin, G. J.; Graves, A. P.; Dill, K. A.; Shoichet, B. K. J. Mol. Biol. 2009, 394, 747.

(24) Fujitani, H.; Tanida, Y.; Ito, M.; Jayachandran, G.; Snow, C. D.; Shirts, M. R.; Sorin, E. J.; Pande, V. S. J. Chem. Phys. 2005, 123, 084108.

(25) Mobley, D. L.; Graves, A. P.; Chodera, J. D.; McReynolds, A. C.; Shoichet, B. K.; Dill, K. A. J. Mol. Biol. 2007, 371, 1118.

(26) Rocklin, G. J.; Boyce, S. E.; Fischer, M.; Fish, I.; Mobley, D. L.; Shoichet, B. K.; Dill, K. A. J. Mol. Biol. 2013, 425, 4569.

(27) Chinea, G.; Padron, G.; Hooft, R. W. W.; Sander, C.; Vriend, G. Proteins: Struct., Funct., Genet. 1995, 23, 415.

(28) Vriend, G. J. Mol. Graphics 1990, 8, 52.

(29) Wang, J.; Wolf, R. M.; Caldwell, J. W.; Kollman, P. A.; Case, D. A. J. Comput. Chem. 2004, 25, 1157.

(30) Bayly, C. I.; Cieplak, P.; Cornell, W.; Kollman, P. A. J. Phys. Chem. 1993, 97, 10269.

(31) Vanquelef, E.; Simon, S.; Marquant, G.; Garcia, E.; Klimerak, G.; Delepine, J. C.; Cieplak, P.; Dupradeau, F.-Y. Nucleic Acids Res. 2011, 39, W511.

(32) Besler, B. H.; Merz, K. M.; Kollman, P. A. J. Comput. Chem. 1990, $11,431$.

(33) Singh, U. C.; Kollman, P. A. J. Comput. Chem. 1984, 5, 129.

(34) Sousa da Silva, A.; Vranken, W. BMC Res. Notes 2012, 5, 367.

(35) Jorgensen, W. L.; Chandrasekhar, J.; Madura, J. D.; Impey, R. W.; Klein, M. L. J. Chem. Phys. 1983, 79, 926.

(36) Lindorff-Larsen, K.; Piana, S.; Palmo, K.; Maragakis, P.; Klepeis, J.; Dror, R.; Shaw, D. Proteins: Struct., Funct., Genet. 2010, 78, 1950.

(37) Picaud, S.; Wells, C.; Felletar, I.; Brotherton, D.; Martin, S.; Savitsky, P.; Diez-Dacal, B.; Philpott, M.; Bountra, C.; Lingard, H.; Fedorov, O.; Müller, S.; Brennan, P. E.; Knapp, S.; Filippakopoulos, P. Proc. Natl. Acad. Sci. U. S. A. 2013, 110, 19754. 
(38) Myszka, D. G.; Abdiche, Y. N.; Arisaka, F.; Byron, O.; Eisenstein, E.; Hensley, P.; Thomson, J. A.; Lombardo, C. R.; Schwarz, F.; Stafford, W.; Doyle, M. L. J. Biomol. Tech. 2003, 14, 247.

(39) Picaud, S.; Leonards, K.; Lambert, J.-P.; Dovey, O.; Wells, C.; Fedorov, O.; Monteiro, O.; Fujisawa, T.; Wang, C.-Y.; Lingard, H.; Tallant, C.; Nikbin, N.; Guetzoyan, L.; Ingham, R.; Ley, S. V.; Brennan, P.; Muller, S.; Samsonova, A.; Gingras, A.-C.; Schwaller, J.; Vassiliou, G.; Knapp, S.; Filippakopoulos, P. Sci. Adv. 2016, 2, e1600760.

(40) Hornak, V.; Abel, R.; Okur, A.; Strockbine, B.; Roitberg, A.; Simmerling, C. Proteins: Struct., Funct., Genet. 2006, 65, 712.

(41) Gerber, P.; Müller, K. J. Comput.-Aided Mol. Des. 1995, 9, 251.

(42) Bush, B. L.; Bayly, C. I.; Halgren, T. A. J. Comput. Chem. 1999, 20, 1495

(43) Betz, R. M.; Walker, R. C. J. Comput. Chem. 2015, 36, 79.

(44) Abraham, M.J.; Murtola, T.; Schulz, R.; Páll, S.; Smith, J. C.; Hess, B.; Lindahl, E. SoftwareX 2015, 1-2, 19.

(45) Pires, D. E. V.; Ascher, D. B. Nucleic Acids Res. 2016, 44, W557.

(46) Berendsen, H. J. C.; Postma, J. P. M.; van Gunsteren, W. F.; DiNola, A.; Haak, J. R. J. Chem. Phys. 1984, 81, 3684.

(47) Goga, N.; Rzepiela, A. J.; de Vries, A. H.; Marrink, S. J.; Berendsen, H. J. C. J. Chem. Theory Comput. 2012, 8, 3637.

(48) Van Gunsteren, W. F.; Berendsen, H. J. C. Mol. Simul. 1988, 1, 173.

(49) Parrinello, M.; Rahman, A. J. Appl. Phys. 1981, 52, 7182.

(50) Chodera, J. D.; Shirts, M. R. J. Chem. Phys. 2011, 135, 194110.

(51) Boresch, S.; Tettinger, F.; Leitgeb, M.; Karplus, M. J. Phys. Chem. B 2003, 107, 9535.

(52) Beutler, T. C.; Mark, A. E.; van Schaik, R. C.; Gerber, P. R.; van Gunsteren, W. F. Chem. Phys. Lett. 1994, 222, 529.

(53) Essmann, U.; Perera, L.; Berkowitz, M. L.; Darden, T.; Lee, H.; Pedersen, L. G. J. Chem. Phys. 1995, 103, 8577.

(54) Páll, S.; Hess, B. Comput. Phys. Commun. 2013, 184, 2641.

(55) Hess, B.; Bekker, J.; Berendsen, H. J. C.; Fraaije, J. G. E. M. J. Comput. Chem. 1997, 18, 1463.

(56) Shirts, M. R.; Chodera, J. D. J. Chem. Phys. 2008, 129, 124105.

(57) Parameswaran, S.; Mobley, D. L. J. Comput.-Aided Mol. Des. 2014, 28,825 .

(58) Klimovich, P.; Shirts, M.; Mobley, D. J. Comput.-Aided Mol. Des. 2015, 29, 397.

(59) Chodera, J. D.; Swope, W. C.; Pitera, J. W.; Seok, C.; Dill, K. A. J. Chem. Theory Comput. 2007, 3, 26.

(60) Efron, B.; Gong, G. Am. Stat. 1983, 37, 36.

(61) Bailey, D.; Jahagirdar, R.; Gordon, A.; Hafiane, A.; Campbell, S.; Chatur, S.; Wagner, G. S.; Hansen, H. C.; Chiacchia, F. S.; Johansson, J.; Krimbou, L.; Wong, N. C. W.; Genest, J. J. Am. Coll. Cardiol. 2010, 55, 2580.

(62) Nikolic, D.; Rizzo, M.; Mikhailidis, D. P.; Wong, N. C.; Banach, M. Expert Opin. Invest. Drugs 2015, 24, 1389.

(63) Ballester, P. J.; Schreyer, A.; Blundell, T. L. J. Chem. Inf. Model. 2014, 54, 944.

(64) Ballester, P. J.; Mitchell, J. B. O. Bioinformatics 2010, 26, 1169.

(65) Zhu, J.; Caflisch, A. J. Med. Chem. 2016, 59, 5555.

(66) Huang, L.; Roux, B. J. Chem. Theory Comput. 2013, 9, 3543.

(67) Hansen, E.; Limé, E.; Norrby, P.-O.; Wiest, O. J. Phys. Chem. A 2016, 120, 3677.

(68) Hudson, B. M.; Nguyen, E.; Tantillo, D. J. Org. Biomol. Chem. 2016, 14, 3975.

(69) Harder, E.; Damm, W.; Maple, J.; Wu, C.; Reboul, M.; Xiang, J. Y.; Wang, L.; Lupyan, D.; Dahlgren, M. K.; Knight, J. L.; Kaus, J. W.; Cerutti, D. S.; Krilov, G.; Jorgensen, W. L.; Abel, R.; Friesner, R. A. J. Chem. Theory Comput. 2016, 12, 281.

(70) Yin, J.; Fenley, A. T.; Henriksen, N. M.; Gilson, M. K. J. Phys. Chem. B 2015, 119, 10145.

(71) Wickstrom, L.; Deng, N.; He, P.; Mentes, A.; Nguyen, C.; Gilson, M. K.; Kurtzman, T.; Gallicchio, E.; Levy, R. M. J. Mol. Recognit. 2016, 29, 10.

(72) Lim, N. M.; Wang, L.; Abel, R.; Mobley, D. L. J. Chem. Theory Comput. 2016, 12, 4620.
(73) Wang, L.; Deng, Y.; Knight, J. L.; Wu, Y.; Kim, B.; Sherman, W.; Shelley, J. C.; Lin, T.; Abel, R. J. Chem. Theory Comput. 2013, 9, 1282.

(74) Wang, L.; Berne, B. J.; Friesner, R. A. Proc. Natl. Acad. Sci. U. S. A. 2012, 109, 1937.

(75) Fennell, C. J.; Wymer, K. L.; Mobley, D. L. J. Phys. Chem. B 2014, 118,6438 .

(76) Boulanger, E.; Huang, L.; MacKerell, A. D., Jr.; Roux, B. Biophys. J. 2016, 110, 646a.

(77) Baker, C. M.; Lopes, P. E. M.; Zhu, X.; Roux, B.; MacKerell, A. D. J. Chem. Theory Comput. 2010, 6, 1181.

(78) Jiao, D.; Golubkov, P. A.; Darden, T. A.; Ren, P. Proc. Natl. Acad. Sci. U. S. A. 2008, 105, 6290.

(79) Duan, L. L.; Feng, G. Q.; Zhang, Q. G. Sci. Rep. 2016, 6, 31488.

(80) Cortopassi, W. A.; Kumar, K.; Paton, R. S. Org. Biomol. Chem. 2016, 14, 10926.

(81) Hay, D. A.; Fedorov, O.; Martin, S.; Singleton, D. C.; Tallant, C.; Wells, C.; Picaud, S.; Philpott, M.; Monteiro, O. P.; Rogers, C. M.; Conway, S. J.; Rooney, T. P. C.; Tumber, A.; Yapp, C.; Filippakopoulos, P.; Bunnage, M. E.; Müller, S.; Knapp, S.; Schofield, C. J.; Brennan, P. E. J. Am. Chem. Soc. 2014, 136, 9308.

(82) Rooney, T. P. C.; Filippakopoulos, P.; Fedorov, O.; Picaud, S.; Cortopassi, W. A.; Hay, D. A.; Martin, S.; Tumber, A.; Rogers, C. M.; Philpott, M.; Wang, M.; Thompson, A. L.; Heightman, T. D.; Pryde, D. C.; Cook, A.; Paton, R. S.; Müller, S.; Knapp, S.; Brennan, P. E.; Conway, S. J. Angew. Chem., Int. Ed. 2014, 53, 6126.

(83) Du, Q.-S.; Long, S.-Y.; Meng, J.-Z.; Huang, R.-B. J. Comput. Chem. 2012, 33, 153

(84) Hu, Y.; Sherborne, B.; Lee, T.-S.; Case, D. A.; York, D. M.; Guo, Z. J. Comput.-Aided Mol. Des. 2016, 30, 533.

(85) Perez, A.; Morrone, J. A.; Simmerling, C.; Dill, K. A. Curr. Opin. Struct. Biol. 2016, 36, 25.

(86) Mobley, D. L.; Gilson, M. K. bioRxiv 2016, 074625.

(87) Steinbrecher, T. B.; Dahlgren, M.; Cappel, D.; Lin, T.; Wang, L.; Krilov, G.; Abel, R.; Friesner, R.; Sherman, W. J. Chem. Inf. Model. 2015, $55,2411$.

(88) Klimovich, P. V.; Mobley, D. L. J. Comput.-Aided Mol. Des. 2015, 29, 1007.

(89) Loeffler, H. H.; Michel, J.; Woods, C. J. Chem. Inf. Model. 2015, 55, 2485.

(90) Homeyer, N.; Gohlke, H. J. Comput. Chem. 2013, 34, 965.

(91) Liu, P.; Dehez, F.; Cai, W.; Chipot, C. J. Chem. Theory Comput. 2012, 8, 2606.

(92) Lundborg, M.; Lindahl, E. J. Phys. Chem. B 2015, 119, 810.

(93) Kumar, A.; Zhang, K. Y. J. Methods 2015, 71, 26.

(94) Genheden, S.; Ryde, U. Expert Opin. Drug Discovery 2015, 10, 449. 